\title{
LINEAR ANALOGUES OF ULTRAFILTERS
}

\author{
BY ALFRED W. HALES ${ }^{1}$
}

Communicated by W. Wistar Comfort, November 24, 1975

1. Introduction. The automorphism group of a vector space $V$ acts on any object constructed naturally from $V$, and the corresponding orbit space is often of considerable mathematical interest. Indeed many classical algebraic questions can be viewed in this way. In this paper we consider the (classically uninteresting) case when the object is the second dual $V^{* *}$ of $V$. The motivation for this is threefold: first, it arises naturally in the above context; second, it is essentially a linear analogue of the study of types of ultrafilters; and third, it has applications to structure theory for infinite abelian groups and primitive rings with minimal left ideals [2].

We present here a statement of some of our results. Details and further results will appear elsewhere.

2. The orbit space. Let $F$ be a field and let $V$ be a vector space over $F$. Then $V$ is naturally embedded in its second dual $V^{* *}$. Furthermore any endomorphism $\varphi: V \rightarrow V$ extends naturally to an endomorphism $\varphi^{* *}: V^{* *} \rightarrow V^{* *}$ Hence the action of the general linear group GL(V) on $V$ extends to an action of $\mathrm{GL}(V)$ on $V^{* *}$, and we can consider the orbit space $V^{* *} / \mathrm{GL}(V)$. If $V$ is finite dimensional, then $V=V^{* *}$ and the orbit space contains just two elements $\bar{o}$ and $\bar{v}$, where "bar" denotes projection and $v$ is any nonzero element of $V$. If $V$ is infinite dimensional, however, then $V^{* *}$ is much larger than $V$ and the orbit space is highly nontrivial.

Henceforth we consider only the case when $F$ is finite and $V$ has countable dimension. (In the applications $F$ is usually a prime field.) Then $V$ has cardinality $\boldsymbol{\kappa}_{0}, V^{*}$ has cardinality $2^{\boldsymbol{\kappa}_{0}}$, and $V^{* *}$ has cardinality $2^{\left(2^{\boldsymbol{N}_{0}}\right)}$. Since GL(V) has cardinality $2^{\boldsymbol{X}_{0}}$ it is immediate that the orbit space has cardinality $2^{\left(2^{\boldsymbol{N}_{0}}\right)}$. We wish to analyze the structure of this orbit space, and in particular to determine its dependence on the field $F$.

3. The partial order. There is a natural partial order on the orbit space $V^{* *} / \mathrm{GL}(V)$ which is analogous to the Rudin-Keisler order on ultrafilters. If $\lambda_{1}$, $\lambda_{2}$ are in $V^{* *}$ we define $\lambda_{1} \geqslant \lambda_{2}$ if and only if $\varphi^{* *}\left(\lambda_{1}\right)=\lambda_{2}$ for some endomorphism $\varphi$ of $V$. The relation $\geqslant$ is clearly reflexive and transitive. Furthermore, if $\lambda_{1}$ and $\lambda_{2}$ lie in the same orbit, then $\lambda_{1} \geqslant \lambda_{2} \geqslant \lambda_{1}$. The converse fails, however, 
so that $\geqslant$ does not quite introduce a partial order on orbits. We do have the following analogue of a standard result on ultrafilters.

THEOREM 1. Suppose that $\lambda \in V^{* *}$ and $\varphi$ is an endomorphism of $V$ with $\varphi^{* *}(\lambda)=\lambda$. Then $\lambda \in W^{* *}$ where $W=\{v: \varphi(v)=v\}$.

Let us define two elements $\lambda_{1}, \lambda_{2} \in V^{* *}$ to be equivalent $\left(\lambda_{1} \equiv \lambda_{2}\right)$ iff $\lambda_{1} \geqslant \lambda_{2} \geqslant \lambda_{1}$. Then we have

COROLlary 2. An equivalence class of $\lambda$ 's is either an orbit or the union of $\boldsymbol{N}_{0}$ orbits (and both cases occur).

We can refine the relation $\geqslant$ by defining $\lambda_{1} \geqslant \lambda_{2}$ if and only if there is an epimorphism $\varphi$ with $\varphi^{* *}\left(\lambda_{1}\right)=\lambda_{2}$. (For equivalent $\lambda_{1}, \lambda_{2}$ this is the same as asking for a monomorphism $\psi$ with $\psi^{* *}\left(\lambda_{2}\right)=\lambda_{1}$.) Then we have

COROLlary 3. If $\lambda_{1} \geqslant \lambda_{2} \geqslant \lambda_{1}$ then $\lambda_{1}$ and $\lambda_{2}$ lie in the same orbit. Furthermore the orbits in a given equivalence class, under the order induced by $\geqslant^{\prime}$, have the order type of either a singleton or of $\mathbf{Z} \cup\{+\infty\}$.

The elements $\bar{o}, \bar{v}$ (where $\bar{o} \leqslant \bar{v}$ ) of $V / \mathrm{GL}(V)$ are dominated by every other orbit in $V^{* *} / \mathrm{GL}(V)$.

4. Ultrafilters. For further analysis of the orbit space we associate to each ultrafilter on a countable set an element of $V^{* *}$. Let $B=\left\{v_{1}, v_{2}, \ldots\right\}$ be a basis of $V$, and let $\beta(B)$ denote the Stone-Čech compactification of $B$, i.e., the collection of ultrafilters $U$ on $B$. Any such $U$ induces an element $\lambda_{U}$ of $V^{* *}$ as follows: if $f$ is in $V^{*}$ then $f$ is constant a.e. (with respect to $U$ ) on $B$, and we define $\lambda_{u}(f)$ to be this constant. Note that the orbit containing $\lambda_{u}$ does not depend on the choice of $B$.

It is easy to see that $\lambda_{U}=\lambda_{V}$ if and only if $U=V$. If $U$ and $V$ are ultrafilters of the same type (i.e. differing by a permutation of $B$ ) then $\lambda_{u}$ and $\lambda_{V}$ lie in the same orbit. We conjecture that the converse is true. This would follow from the more general conjecture (whose converse is true):

Conjecture 4. If $\lambda_{U} \geqslant \lambda_{v}$ then $U$ dominates $V$ in the Rudin-Keisler ordering of ultrafilters [1].

We can prove this in various special cases, such as the case where $U$ is a Ramsey ultrafilter.

The $\lambda_{u}$ do not exhaust $V^{* *}$, and in fact not every orbit contains a $\lambda_{u}$. If we denote by $L_{1}$ the collection of orbits containing a $\lambda_{u}$, by $L_{2}$ those containing a $\left(\lambda_{u}+\lambda_{v}\right)$, etc, then we have

THEOREM 5. The sequence $L_{1} \subseteq L_{2} \subseteq L_{3} \ldots$ is strictly increasing and its union is a proper subset of $V^{* *} / \mathrm{GL}(V)$.

Although not every element of $V^{* *}$ comes from an ultrafilter on $B$, the following theorem essentially says that every one comes from an ultrafilter on $V$ : 
THEOREM 6. For every $\lambda$ in $V^{* *}$ there exists $U$ in $\beta(B)$ with $\lambda_{U} \geqslant \lambda$.

In other words, the $\lambda_{u}$ are cofinal. In contrast to this we have

THEOREM 7. There exist $\lambda$ in $V^{* *}-V$ such that $\lambda \ngtr \lambda_{u}$ for any $U$ in $\beta(B)-B$.

Finally we have

THEOREM 8. If $U$ is a Ramsey ultrafilter then $\lambda_{U}$ is minimal in $\left(V^{* *}-V\right) / \mathrm{GL}(V)$

5. Conclusion. If Conjecture 4 holds then the types of ultrafilters in $\beta(B)$ provide invariants for $L_{1}$, and in addition establish a natural 1-1 correspondence between the $L_{1}$ as the field $F$ varies. It should be possible to extend this to the higher levels $L_{n}, n \geqslant 2$. For the remaining orbits, i.e. those not in any $L_{n}$, a different approach will probably be needed.

There are in addition many open (technical) questions involving the partial order on the orbits.

\section{REFERENCES}

1. W. W. Comfort and S. Negrepontis, The theory of ultrafilters, Die Grundlehren der math. Wissenschaften, Band 211, Springer-Verlag, Berlin and New York, 1974.

2. A. W. Hales, Ultrafilters and abelian groups, Bull. Amer. Math. Soc. (to appear). $353-362$.

3. M. E. Rudin, Partial orders on the types in $\beta N$, Trans. Amer. Math. Soc. 155 (1971),

DEPARTMENT OF MATHEMATICS, UNIVERSITY OF CALIFORNIA, LOS ANGELES, CALIFORNIA 90024 